\title{
A la recherche de l'efficacité
}

Financement et réformes dans l'enseignement supérieur en Suisse

A search for efficiency. Fundingand and reforms in higher education in Switzerland

En búsqueda de la eficacia. Financiación y reformas en la enseñanza superior en Suiza

\section{Edo Poglia}

\section{OpenEdition \\ Journals}

Édition électronique

URL : http://journals.openedition.org/ries/2993

DOI : 10.4000/ries.2993

ISSN : 2261-4265

Éditeur

Centre international d'études pédagogiques

Édition imprimée

Date de publication : 1 juin 1999

Pagination : 93-103

ISSN : 1254-4590

Référence électronique

Edo Poglia, «A la recherche de l'efficacité », Revue internationale d'éducation de Sèvres [En ligne],

22 | 1999, mis en ligne le 13 mai 2013, consulté le 01 mai 2019. URL : http://journals.openedition.org/ ries/2993; DOI : 10.4000/ries.2993

Ce document a été généré automatiquement le 1 mai 2019.

(c) Tous droits réservés 


\section{A la recherche de l'efficacité}

Financement et réformes dans l'enseignement supérieur en Suisse

A search for efficiency. Fundingand and reforms in higher education in

Switzerland

En búsqueda de la eficacia. Financiación y reformas en la enseñanza superior en Suiza

\section{Edo Poglia}

\section{Fédéralisme, décentralisation et ( ?) autonomie}

1 La politique éducative suisse est fortement marquée par le principe de base qui régit l'État suisse : celui du fédéralisme, entendu dans ce pays comme la volonté de garder un pouvoir décisionnel fort au niveau des vingt-six mini-États qui constituent la Confédération helvétique : l'éducation est, en effet, le secteur où l'autonomie des cantons par rapport à la Confédération s'exerce de la manière la plus évidente. En outre, plusieurs de ces cantons partagent une partie de leur pouvoir éducatif - mais aussi des charges financières - 94 avec les communes qui les composent.

2 Ainsi, constitutionnellement, la Confédération ne peut intervenir que dans certains secteurs de la formation, notamment dans la formation professionnelle, qu'elle soit de base ou supérieure et, en partie, dans les formations médicales et la " maturité ${ }^{1}$ ».

3 Si l'intervention de l'État central est assez marquée au niveau de l'enseignement supérieur - avec la prise en charge de la moitié du financement, elle n'en reste pas moins assez strictement délimitée à la gestion directe des deux Écoles polytechniques, qui forment les ingénieurs universitaires et un bon nombre de scientifiques, et au subventionnement des huit universités cantonales ${ }^{2}$ (plus deux en développement).

4 Seule la politique de la recherche, y compris la recherche universitaire - qui constitue néanmoins en Suisse, plus qu'ailleurs, une part importante de toute la recherche publique - est pleinement du ressort fédéral. 
5 Le débat "public ou privé " n'est pour l'instant pas très actuel dans le domaine de l'éducation, même si le libéralisme économique est fortement ancré en Suisse et qu'on y procède, comme ailleurs, à des privatisations, du moins partielles, dans des secteurs économiques comme celui des télécommunications.

6 La répartition de la charge budgétaire offre un bon reflet de la répartition des tâches et du pouvoir éducatif en Suisse :

Dépenses publiques

\begin{tabular}{|l|c|}
\hline \multicolumn{2}{|c|}{$\begin{array}{c}\text { Dépenses publiques pour l'enseignement (1996) } \\
\text { (en milliards de francs suisses) }\end{array}$} \\
\hline Total & 20,7 \\
\hline Confédération & 2,5 \\
\hline Cantons & 10,8 \\
\hline Communes & 7,4 \\
\hline \multicolumn{2}{|c|}{ Dépenses publiques pour les Hautes écoles (1996) ${ }^{4}$} \\
(en milliards de francs suisses) \\
\hline Total & 3,53 \\
\hline Confédération & 1,78 \\
\hline Cantons & 1,73 \\
\hline Communes & 0,014 \\
\hline
\end{tabular}

Office fédéral de la statistique (OFS), Annuaire statistique de la Suisse, Neuchâtel, 1999.

7 La problématique de la décentralisation, en éducation comme dans d'autres domaines, présente deux facettes que le débat politique confond souvent et volontiers: décentralisation étatique, d'une part (en Suisse, de la Confédération aux cantons et des cantons aux communes); décentralisation institutionnelle, de l'autre, impliquant une capacité d'autogestion de l'établissement éducatif, par exemple de l'université et de ses membres, dans le cadre d'une enveloppe financière accordée par l'État (pour ce qui touche aux choix des filières de formation offertes, aux priorités de recherche, à la politique du personnel, etc.).

$8 \mathrm{Si}$, pour la formation obligatoire et pour celle du secondaire supérieur, la décentralisation institutionnelle est assez limitée en Suisse (concernant notamment le choix des programmes scolaires, des moyens d'enseignement, des normes de sélection), la situation est plus complexe pour la formation universitaire. D'une part, l'État cantonal est traditionnellement nanti de compétences importantes, telles que la nomination des professeurs qui acquièrent un statut analogue aux autres fonctionnaires de l'État, de l'autre, les professeurs, individuellement ou au niveau universitaire, jouissent d'une très large autonomie scientifique et didactique.

9 En revanche, on peut admettre que le pouvoir et la capacité de gestion de « l'institution université » dans son ensemble, exercés par exemple par les rectorats, est plutôt faible, avec une notable exception pour les Écoles polytechniques. 


\section{Problèmes d'efficacité et d'efficience}

\section{Efficacité}

10 Si l'on définit l'efficacité, dans ce domaine, comme la capacité du système de formation supérieure d'atteindre les objectifs qu'il s'est fixé ou qu'on lui a fixés, la première difficulté à laquelle est confrontée l'observateur, et qui est liée à la structure éducative suisse décrite précédemment, est de comprendre quels sont réellement les « objectifs de l'université » et qui les définit. L'État cantonal en fixe certains, mais de manière très sommaire, dans le cadre des lois ou des «messages» qui accompagnent les demandes de budget aux Parlements cantonaux. L'institution universitaire elle-même - de par la faiblesse traditionnelle de sa gestion et de sa planification centrale - ne le fait pas de manière beaucoup plus précise et, en tout cas, est peu portée à les rendre opérationnels.

Diverses options et opinions coexistent donc, même sur des sujets fondamentaux comme les aspects quantitatifs (évolution souhaitée du nombre d'étudiants) ou les développements sectoriels et disciplinaires à privilégier. Faut-il favoriser certaines disciplines et les développer autour de centres d'excellence existants ou, au contraire, rééquilibrer les différents domaines du point de vue de leur dotation pour la recherche ou des taux d'encadrement des étudiants ? Certains, par exemple, plaident en faveur d'un rééquilibrage au profit des sciences sociales et économiques «surchargées » du point de vue du nombre des étudiants.

Il va de soi que, si des objectifs communs ne sont pas faciles à déceler dans le cadre de chaque université prise individuellement, ils ne peuvent pas l'être beaucoup plus aisément pour le "système » universitaire suisse dans son ensemble. Ceci est d'autant plus vrai que, comme il a été rappelé plus haut, la Confédération n'a que relativement peu de pouvoir et que la coordination interuniversitaire n'est que peu développée, tant entre les instances politiques (cantons) qu'entre les rectorats. Le fait que deux " poids lourds » universitaires - les Écoles polytechniques - soient de compétence fédérale ne simplifie d'ailleurs pas le paysage et la coordination universitaire suisse.

13 L'introduction récente du numerus clausus à l'entrée des études de médecine - adopté après maints tiraillements et d'ailleurs seulement dans une partie des universités du pays - est éclairante à ce sujet.

14 Ainsi, plus que « d'objectifs » atteints plus ou moins efficacement par les universités, on peut parler de "thèmes névralgiques" autour desquels s'articule, depuis quelques années, le débat sur la politique universitaire. Évoquons les principaux d'entre eux.

15 La part de la population étudiante est, en Suisse, assez modeste si on la compare à celle qu'on peut observer au niveau international ${ }^{3}$. Si cette donnée réjouit ceux qui redoutent le spectre de l'« université de masse », dépourvue de qualité scientifique et pédagogique, elle déclenche les critiques de ceux qui, soucieux d'égalisation des chances pour les femmes ainsi que pour les régions et les catégories sociales économiquement plus faibles, sont préoccupés par un certain malthusianisme scientifique qui risquerait de dégarnir le futur marché du travail, censé être toujours plus demandeur de compétences de haut niveau. Les défenseurs du maintien restrictif à l'université ont été confortés par des analyses récentes mettant en lumière le fait que, déjà actuellement, les revenus supplémentaires individuels engendrés par un diplôme universitaire ont tendance à être 
moins importants en Suisse que dans plusieurs autres pays, comme les États- Unis, l'Angleterre, la France et l'Allemagne. Par contre, la situation des diplômés du tertiaire est très satisfaisante à ce point de vue ${ }^{4}$. Comparativement aux autres formations, la formation universitaire "protège " d'ailleurs plutôt moins bien du chômage que dans d'autres pays (même si les taux de chômage globaux sont assez bas en Suisse).

L'adéquation entre le choix de la discipline de formation supérieure opéré par les jeunes et le marché du travail fait problème aux yeux de certains critiques mais, là aussi, les opinions ne concordent pas toujours. Si des secteurs économiques comme l'informatique sont fortement demandeurs de diplômés - que l'université peine à former en nombre voulu -, les statistiques de l'emploi des jeunes diplômés montrent des situations assez différenciées en fonction des disciplines étudiées. Les sciences sociales, les lettres et les sciences de l'ingénieur n'offrent pas les mêmes débouchés que la médecine ou le droit ${ }^{5}$. Les critiques concernant les « compétences réellement acquises » ne manquent pas - ainsi les doléances visant le manque d'esprit d'entreprise et d'innovation ou l'insuffisante disponibilité à la mobilité géographique et linguistique - mais ces critiques pourraient aussi n'être qu'un exercice « de routine " propre à chaque système de formation.

Des critiques concernant la « qualité » de la formation universitaire en général font partie du paysage presque normal du débat dans ce domaine, la Suisse n'y échappe pas. Les quelques données disponibles donnent néanmoins à penser que, si des problèmes réels existent, ils consistent plutôt à rechercher les aspects pédagogiques et didactiques que les contenus scientifiques et professionnels ${ }^{6}$. Par contre, les critiques visant le peu d'empressement des professeurs à offrir des approches interdisciplinaires se font de plus en plus entendre.

18 Étant donné la structure et l'organisation universitaires très diversifiées des études, les universités suisses ont de la peine à adopter le système plus flexible des crédits transférables. Cette absence de souplesse ne favorise d'ailleurs pas la mobilité interuniversitaire, ni probablement la «saine concurrence» entre institutions de formation, invoquée par certains comme moyen de dynamiser le paysage universitaire suisse.

19 Si l'égalité des chances entre les filles et les garçons d'accéder à une formation universitaire tend à être établie (et elle l'est déjà dans la partie francophone du pays), tel n'est pas le cas en ce qui concerne la provenance sociale ou géographique. Face à des taux d'entrée à l'université supérieurs à $25 \%$ dans le canton de Genève, on trouve presque une dizaine d'autres cantons avec des taux se situant autour de $10 \%$ ou moins ${ }^{7}$.

Les objectifs fixés au système universitaire suisse du point de vue de la recherche scientifique sont par contre assez largement atteints. Non seulement les universités réalisent une part de recherche fondamentale nationale comparativement plus importante que dans beaucoup de pays, mais, de plus, leurs résultats sont internationalement excellents - par exemple, en termes de publications et citations dans plusieurs grands domaines des sciences naturelles, exactes, médicales et techniques (immunologie, physique, biologie moléculaire, etc.). Avec 17,4 publications scientifiques pour 1000 habitants, la Suisse se plaçait par exemple de 1981 à 1995 au premier rang mondial et ceci est aussi vrai pour l'impact de ces publications mesuré à l'aune des citations $^{8}$.

21 Pour résumer, on peut donc affirmer que du point de vue de l'efficacité, le système universitaire suisse est loin d'être aussi grevé de problèmes que certains de ses critiques 
le prétendent. Par contre, l'absence d'une définition claire et cohérente des objectifs à atteindre peut paraître étonnante. Mesurer l'efficacité dans cette situation devient donc réellement une gageure.

\section{Efficience}

22 La définition d'efficience - entendue comme l'emploi optimal des ressources, notamment financières, pour atteindre les résultats escomptés - glisse souvent, dans le discours politique et administratif courant, vers l'idée de minimisation des ressources, de coupures budgétaires et vers la formule un peu velléitaire de " faire mieux avec moins ».

Si le sentiment assez largement partagé par les milieux politiques suisses par rapport à la formation supérieure est que l'efficience n'est pas toujours au rendez-vous, la quantification de ce sentiment n'est ni aisée ni univoque. Voici quelques uns des aspects du débat actuel sur l'efficience dans les milieux de la politique universitaire.

Avec des dépenses et des coûts de fonctionnement du système universitaire suisse (huit universités cantonales et deux Écoles polytechniques fédérales) qui s'établissent à presque quatre milliards de francs suisses par an, la part des dépenses publiques directes en faveur des établissements tertiaires rapportée au PIB se situe, au niveau international, légèrement au-dessus de la moyenne des pays de l'OCDE (1,1 \% en 1998 par rapport à 0,9\% en 1995). Par contre, la dépense par étudiant dans les établissements tertiaires, avec l'équivalant de 16000 dollars, représente le double de la dépense moyenne pour les pays de l'OCDE et se place au deuxième rang international de ces dépenses?

$\mathrm{Au} \mathrm{vu}$ de ces derniers chiffres, la formation universitaire suisse serait donc soit relativement efficiente, soit très peu efficiente. La question qui se pose est évidemment celle des références et des définitions d'efficience dans ce cas précis ${ }^{10}$. Il va de soi que dans le débat politico-médiatique l'une ou l'autre de ces références est employée selon les besoins de la démonstration.

D'autres observations, ou parfois des «impressions ", mettent en cause l'efficience de la formation universitaire suisse. Ainsi, la coordination insuffisante entre les diverses institutions universitaires du pays est souvent mise en cause dans la mesure où elle serait source de doubles emplois et du maintien (coûteux) de filières de formation sans la masse nécessaire suffisante. On critique aussi les différences de rémunération entre les professeurs des diverses universités, pouvant atteindre un quart du salaire, sans différences de prestation.

On souligne par ailleurs les différences entre les taux d'encadrement des diverses disciplines et établissements : environ soixante à soixante-dix étudiants par professeur en sciences sociales, contre environ vingt en médecine ou en théologie ${ }^{11}$. Ces différences sont jugées insupportables par les "défavorisés ", mais d'un certain point de vue - qui est très contesté-elles pourraient être considérées comme plus efficientes. On souligne aussi les différences très marquées entre nombre de diplômés par professeur entre disciplines (presque vingt en 1995 pour la politologie contre moins de deux en mathématiques, physique, linguistique, etc. ${ }^{12}$. À l'inverse, on fait remarquer que la production scientifique est, elle, bien plus visible et consistante dans les facultés des sciences naturelles ou médicales que dans celles des sciences humaines et sociales.

La durée réputée globalement trop longue des études (5,9 années) et ses variations selon les disciplines et les établissements sont sources de discussions (de plus de quatre années 
pour le droit à Lausanne à huit années pour les sciences humaines et sociales à Berne) ${ }^{13}$. Un lien éventuel entre durée et coût par étudiant, les deux étant trop élevés, suscite également des suspicions.

Comme on peut le constater, ces données et ces critiques sectorielles ne fournissent pas encore une réponse claire à la question de l'efficience réelle du système universitaire suisse ni, $a$ fortiori, à la question des facteurs d'efficience ou d'inefficience. Ceci est vrai tant pour ce qui concerne son efficience d'ensemble, par rapport aux grandes écoles étrangères, que pour ce qui concerne la comparaison interne entre l'efficience de ses établissements et de ses composantes disciplinaires.

Pour fournir au moins un début de réponse sur les aspects comparatifs internes, nous avons voulu, dans un premier temps, porter l'attention sur l'efficience des instituts universitaires dans le domaine de la recherche en sciences naturelles et en sciences médicales. Nos recherches montrent que - contrairement à certaines hypothèses «politiques »- la corrélation entre outputs (résultats de recherche mesurés par les publications $\mathrm{SCI}$ ) et inputs financiers est plutôt bonne et qu'il ne semble pas fondé, à la lumière de ces données, de parler de différences vraiment marquantes d'efficience de recherche entre instituts ${ }^{14}$.

Tableau 1

Output (publications scientifiques, 1994)

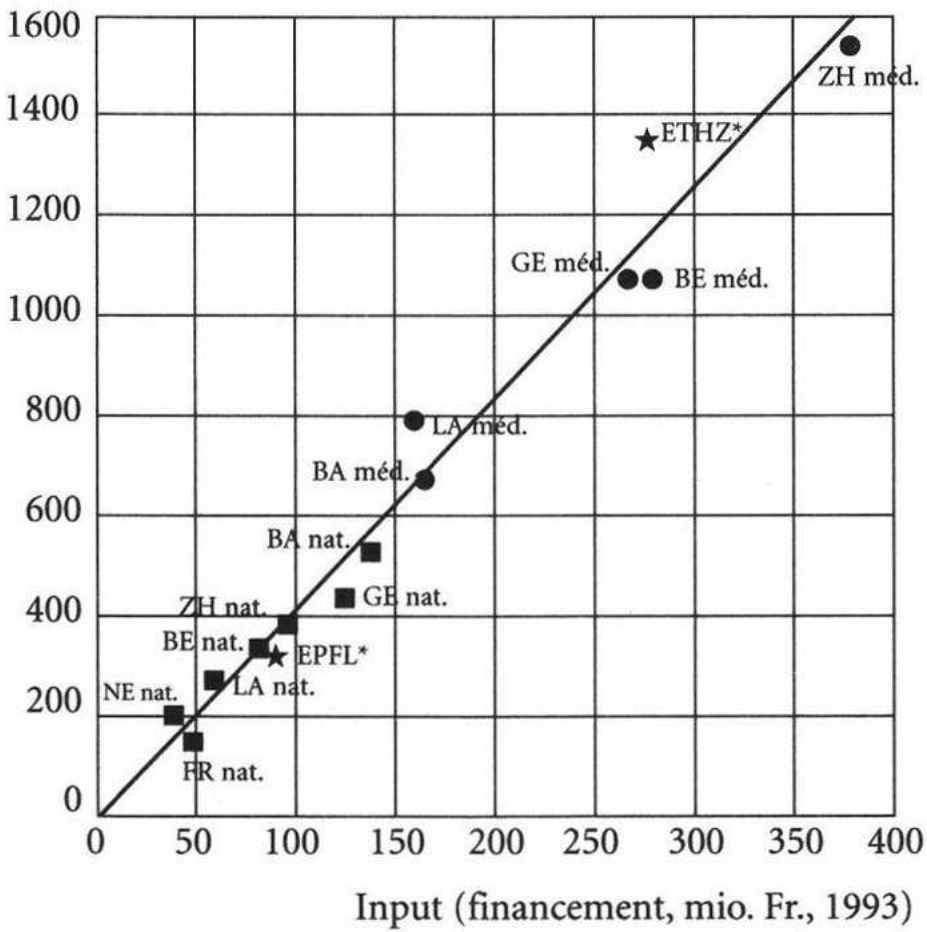

méd. : Faculté de médecine et hôpitaux universitaires nat. : Faculté de sciences exactes et naturelles * seulement départements de sciences exactes et naturelles

31 La question de l'efficience universitaire touche aussi, ou peut-être surtout, l'enseignement et non pas seulement la recherche. Pour essayer de répondre aux interrogations sur l'efficience globale (formation et recherche), nous avons donc tenté, dans un deuxième temps, de construire des indicateurs d'output en tenant compte des deux aspects, ceci en compactant les résultats de recherche (indicateur: nombre de 
publications) et les résultats de formation (indicateur : nombre de diplômes). Du côté des inputs nous avons considéré soit les charges en personnel (professeurs, autres enseignants du niveau supérieur et assistants, cf. tableau 2), soit directement les charges financières (cf. tableau 3) ${ }^{15}$.

Les résultats, pour l'instant très provisoires, sont plutôt surprenants vu les critiques rappelées supra. La corrélation - rapportée tant aux établissements universitaires qu'aux disciplines scientifiques pour l'ensemble du pays - entre inputs (personnel ou finances) et outputs (diplômes et résultats de recherche) sont en effet remarquables, ce qui tendrait à exclure des chutes d'efficience macroscopiques dans certains établissements ou certaines disciplines.

\section{Tableau 2}

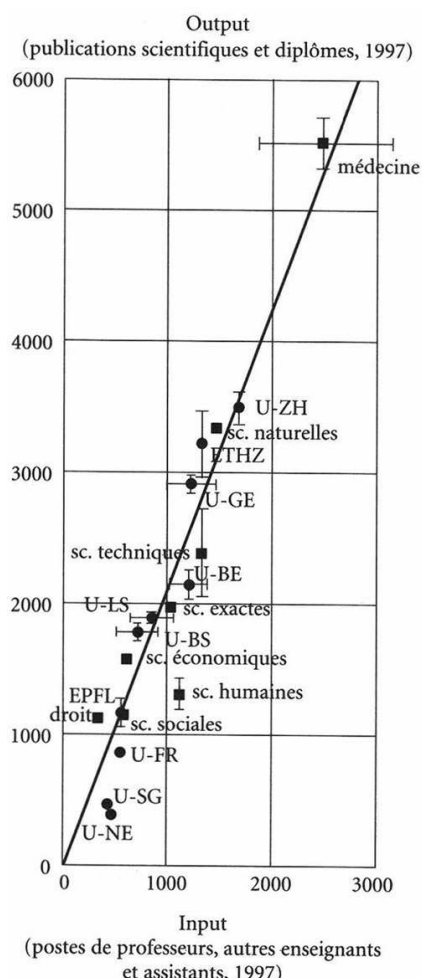




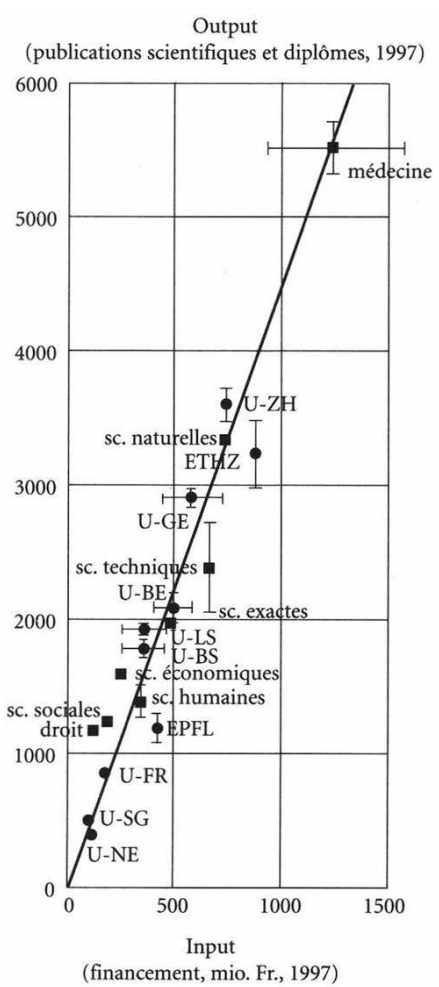

\section{Les politiques universitaires actuelles}

La question de l'efficience (parfois désignée avec d'autres termes) ayant beaucoup préoccupé les milieux politiques suisses ces dernières années, plusieurs « remèdes » ont donc été proposés et certains adoptés pour pallier les problèmes réels ou supposés, et ceci tant aux niveaux des divers cantons universitaires que de la Confédération suisse.

Ainsi, des lois cantonales ont été révisées dans le but notamment de renforcer le pouvoir d'un rectorat censé mieux assurer son mandat de " gestion efficiente » de l'université, ou dans le but d'accorder à l'université plus d'autonomie par rapport à l'État (université de Bâle). Un pas supplémentaire en vue de renforcer l'autonomie - déjà importante - des Écoles polytechniques a aussi été réalisé avec la révision de la loi fédérale correspondante.

Une (certaine) autonomie budgétaire fait partie de ces innovations, timide encore pour les universités cantonales, importante pour les Écoles polytechniques. D'autre part, les cantons non universitaires se sont engagés, dans le cadre d'un concordat intercantonal, à financer les cantons qui gèrent une université sur la base du nombre d'étudiants qu'ils y envoient.

Des efforts - suscitant parfois des débats houleux - sont en cours en vue de créer des synergies et des collaborations entre universités (enseignements en commun), de réaliser une meilleure répartition des tâches de formation et de recherche (notamment entre les universités de Genève et de Lausanne et l'École polytechnique fédérale de Lausanne). On évoque même de possibles fusions entre universités. 
37 Avec l'assentiment de la Confédération qui le finance, le Fonds national suisse de la recherche scientifique a, de son côté, lancé un programme de construction et financement de "pôles de recherche " sectoriels, comprenant un réseau d'instituts de divers universités autour d'une leading house située dans l'une ou l'autre d'entre elles.

Le gouvernement fédéral a récemment soumis au Parlement une révision assez importante de la loi fédérale d'aide aux universités cantonales qui prévoit, d'une part, un nouveau mode de (co)financement fédéral, lié aux outputs tant de la formation que de la recherche (diplômes et mandats de recherche) et, d'autre part, le renforcement de l'instance politique de coordination entre les cantons universitaires (Conférence universitaire suisse) ainsi que la mise sur pied d'une instance "pour l'assurance et l'évaluation de la qualité universitaire ».

39 Par ailleurs, les nouvelles « Hautes Écoles spécialisées » (universités professionnelles à l'image des Fachhochschulen allemandes), créées en 1996, sont subventionnées par la Confédération selon le critère du nombre d'étudiants et il est prévu d'ajouter bientôt le critère des mandats extérieurs de recherche et développement.

La philosophie universitaire qui occupe actuellement le devant de la scène politique et médiatique est par ailleurs celle du « réseau universitaire suisse », cette idée de réseau se voulant une réponse au souci d'efficience et d'efficacité tout en sauvegardant l'autonomie cantonale en matière de formation supérieure.

41 Le rapide tour d'horizon des problèmes d'efficacité de l'université suisse nous a permis à la fois de mettre en lumière le fait que certains objectifs n'ont pas (encore ?) été suffisamment atteints, mais aussi de montrer que la définition même de ces objectifs reste imprécise et peu concrète. Nous avons aussi tenté de montrer que les critiques et les considérations des insiders universitaires et, spécialement, des milieux politiques, sur le manque d'efficience du système universitaire suisse ne sont, pour l'instant du moins, que partiellement vérifiées par des données quantitatives. Cela n'a pas empêché les milieux politiques de mettre en œuvre, ou en tout cas, de proposer des réformes d'une certaine ampleur, visant un système universitaire plus efficient et pourquoi pas, plus efficace.

Notre conclusion (optimiste et pessimiste à la fois) est peut-être surprenante pour des scientifiques habitués à critiquer la lenteur de la politique à trouver des solutions aux problèmes que leurs analyses auraient découverts et décortiqués. Dans la réalité considérée, ici en l'occurrence celle de l'université suisse, l'analyse scientifique de la situation n'a pas vraiment précédé l'élaboration des politiques inhérentes. Il n'a donc nullement été nécessaire que l'existence des problèmes ait été (scientifiquement) démontrée préalablement pour que le monde politique veuille et puisse s'atteler à les résoudre.

\section{NOTES}

1. Maturité : diplôme de fin d'études secondaires correspondant au niveau du baccalauréat. 
2. Formant en tout environ 90000 étudiants. La plus grande université (Zurich) compte environ 16000 étudiants, la plus petite (Neuchâtel) 3000 et les deux Écoles polytechniques 11500 et 4400 .

3. Le taux d'accès à l'enseignement supérieur universitaire est d'environ $17 \%$ (0FS, Indicateurs des Hautes Écoles suisses, Berne, 1997) contre environ 27 \% en Allemagne et plus de 30 \% aux PaysBas, au Danemark et dans d'autres pays (OCDE, Regards sur l'éducation, Paris, 1998).

4. OFS, Les indicateurs du capital humain, Neuchâtel, 1998 et S. Wolter, B. Weber, « Der monetäre Nutzen von Bildung ", Die Volkswirtschaft, n 9, 1998.

5. OFS, La situation des diplômés universitaires sur le marché de l'emploi en 1997, Neuchâtel, 1998.

6. Par exemple, concernant l'École polytechnique de Zurich, A. Leu et al., Die Wirksamkeit der Ingenieurausbildung in der Schweiz, Chur, Rüegger, 1996.

7. OFS, Indicateurs des Hautes études suisses, Berne, 1997.

8. Schweizerischer Wissenschaftsrat (SWR), Beitrag $z \mathfrak{u}$ einem Indikatorensystem für die Wissenschafts - und Technologiepolitik, Berne, F\&B, 1//99.

9. OCDE 1998, op. cit, note 3 .

10. Ibid. note 8.

11. CSS, Grandes orientations pour le développement des universités et Hautes Écoles suisses 2000-2003, Berne, FS15b, 1997.

12. SWR, Evaluation des Geisteswissenschaftiche Forschung in der Schweiz, Berne, 1998.

13. OFS, Indicateurs des Hautes Écoles suisses, Berne, 1997, pour la cohorte 1980.

14. Op. cit. note 7 .

15. Calculs: M. Von Ins, CSS. Les barres horizontales et verticales indiquent les limites de précision des données.

\section{RÉSUMÉS}

Dans un paysage éducatif complexe où le pouvoir se partage entre État fédéral, cantons et communes, la Suisse n'échappe pas au débat sur la qualité de son enseignement supérieur. L'approche économique de ce système, en terme d'efficacité et d'efficience, conduit l'auteur à des conclusions plutôt optimistes, même si certains objectifs ne sont pas atteints. Les critiques, justifiées ou non, amènent les politiques à s'impliquer en proposant des réformes qui, on peut l'espérer, contribueront à améliorer le fonctionnement de cet enseignement.

In a complex educational system, in which power is shared between the federal State, the regions, and the local authorities, Switzerland still needs to debate on the quality of its higher education system. The economic approach to this system, in terms of effectiveness and efficiency, leads the writer to fairly optimistic conclusions, even if some of the objectives are not attained. Criticism, whether it be justified or not, has led politicians to become involved and propose reforms which, it may be hoped, will help to improve the functioning of this teaching.

En un paisaje educativo complejo en el que Estado Federal, cantónes y municipios se comparten el poder, Suiza no se libra del debate acerca de la calidad de su enseñanza superior. El enfoque económico de este sistema, en términos de eficacia y de eficiencia, conduce al autor a conclusiones más bien optimistas, aunque no se hayan alcanzado algunas metas. Las críticas, sean 
o no justificadas, llevan a los políticos a comprometerse proponiendo reformas que, confiemos en ello, contribuirán a mejorar el funcionamiento de esta docencia.

INDEX

Mots-clés : enseignement supérieur, financement, politique éducative, réforme de l'enseignement

Index géographique : Europe

\section{AUTEUR}

EDO POGLIA

Professeur, Conseil suisse de la science, Berne, Suisse. 